\title{
Ion assisted deposition of thin films by substrate tuned radio frequency magnetron sputtering
}

\author{
A. Lousa ${ }^{\text {a) }}$ and S. Gimeno \\ Departament de Física Aplicada i Electrònica, LFCF, Universitat de Barcelona, Av. Diagonal 647, \\ E 08028 Barcelona, Catalunya, Spain
}

(Received 18 June 1996; accepted 25 October 1996)

\begin{abstract}
The substrate tuning technique was applied to a radio frequency magnetron sputtering system to obtain a variable substrate bias without an additional source. The dependence of the substrate bias on the value of the external impedance was studied for different values of chamber pressure, gas composition and rf input power. A qualitative explanation of the results is given, based on a simple model, and the role of the stray capacitance is clearly disclosed. Langmuir probe measurements show that this system allows independent control of the ion flux and the ion energy bombarding the growing film. For an argon flow rate of $2.8 \mathrm{sccm}$ and a radio frequency power of $300 \mathrm{~W}$ (intermediate values of the range studied) the ion flux incident on the substrate was $1.3 \times 10^{20} \mathrm{~m}^{-2} \mathrm{~s}^{-1}$. The maximum ion energy available in these conditions can be varied in the range $30-150 \mathrm{eV}$. As a practical application of the technique, $\mathrm{BN}$ thin films were deposited under different ion bombardment conditions. An ion energy threshold of about $80 \mathrm{eV}$ was found, below which only the hexagonal phase was present in the films, while for higher energies both hexagonal and cubic phase were present. A cubic content of about $60 \%$ was found for an ion energy of $120 \mathrm{~V}$.

(C) 1997 American Vacuum Society. [S0734-2101(97)03401-5]
\end{abstract}

\section{INTRODUCTION}

The effects of ion bombardment during the growth of thin films has been widely reported. ${ }^{1}$ It changes the properties of thin films, such as morphology, composition, structure, stress, electrical conductivity, and others. Various mechanisms have been proposed to account for these effects: ion implantation, preferential sputtering, transfer of momentum or energy from ions to growing film. These effects strongly depend on the intensity of bombardment which, in turn, is determined by both the ion flux and the ion energy. Moreover, a critical value of the ion/atom arrival rate ratio, which depends on ion energy, has often been found to improve the properties of the films, such as stress relief in evaporated $\mathrm{Ge}$ films, ${ }^{1}$ hardness of carbon nitride coatings ${ }^{2}$ or the synthesis of the BN cubic phase. ${ }^{3}$ Therefore, an accurate control of bombardment intensity is needed in order to deposit films with the desired properties.

In addition to techniques in which an ion beam is directed at the growing film, the deposition methods based on plasmas involve varying intensities of ion bombardment of the substrate. Even when the substrate is grounded, a certain degree of ion bombardment is present as the plasma potential is always positive in relation to ground. When an ionassisted deposition is desired, a negative dc bias voltage need to be developed at the substrate electrode. For nonconducting materials the dc bias voltage is commonly supplied by an additional rf source. But there is a simple alternative called "substrate tuning." This method, first developed by Logan ${ }^{4}$ in a rf diode sputtering system, is based on an adjustable external impedance between the substrate

\footnotetext{
*Published without author corrections.

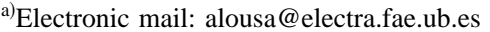

holder and ground. As a consequence, the flow of rf current through the substrate varies, which induces a controlled dc bias potential at the film surface.

Magnetron sputtering has become one of the most widely used techniques in both research and production of thin films. ${ }^{5}$ In a rf magnetron sputtering system, the discharge is confined close to the cathode surface by the magnetic field. This allows work at lower pressures, which makes the sputtering of the target more effective and increasing the growth rate. However, as the discharge is confined close to the target, a less dense plasma is located close to the substrate electrode, resulting in a low ion bombardment there. But if the magnetron cathode is not completely balanced, the plasma region extends along the axis towards the substrate. ${ }^{6}$

In this article we present the results of the substrate tuning technique applied to a rf magnetron sputtering system. The dependence of the substrate bias on the external impedance is studied for different values of chamber pressure, gas composition, and rf input power. The ability of this system to regulate the ion bombardment on the substrate is discussed. We also present the results of depositing boron nitride (BN) thin films in different bombardment conditions using this technique.

\section{EXPERIMENT}

The sputter chamber consisted of a cylindrical vessel with a diameter of $40 \mathrm{~cm}$. A base pressure of about $10^{-4} \mathrm{~Pa}$ was established in the chamber before the sputtering started. Two independent mass flow controllers were used to regulate argon and nitrogen flows. The total gas flow was varied between 1.4 and $5.6 \mathrm{sccm}$, which corresponds to a variation of the total pressure between $5 \times 10^{-2} \mathrm{~Pa}$ and $2 \times 10^{-1} \mathrm{~Pa}$.

Figure 1 shows a diagram of our experimental system. An US'GUNII magnetron cathode was placed near the center of 


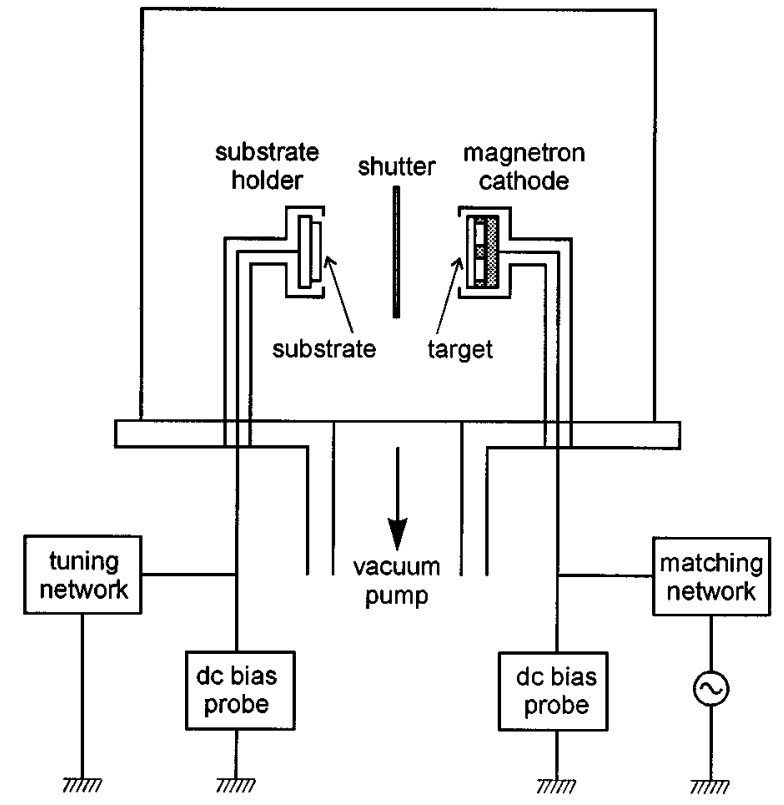

FIG. 1. Diagram (not scaled) of the experimental system.

the chamber with a nonconducting target (hexagonal BN) 3 in. in diameter, and connected to a rf power source through an automatic matching network. The parallel magnetic field measured in the erosion zone on the target was $37 \mathrm{mT}$. On the substrate surface the magnetic field was perpendicular, with an intensity of $3.5 \mathrm{mT}$. This situation corresponds to a somewhat "unbalanced" magnetron, ${ }^{7}$ in which some of the field lines from the annular magnet do not pass into the cylindrical central magnet.

The substrate holder was placed $4 \mathrm{~cm}$ from the cathode, parallel to it, and had a ground shield aperture similar to that of the target. An external tuning device, consisting of a fixed inductor and a variable blocking capacitor in series, was placed between the substrate holder and ground in order to obtain a variable plasma-to-ground impedance. Polished low doped Si crystalline (100) wafers and Corning 7059 glass were used as substrates. The fixed deposition conditions are shown in Table I, the substrate self-bias being the variable parameter. Film composition was determined from electron probe microanalysis (EPMA) measurements, and film structure was studied by Fourier transform infrared absorption spectroscopy (FTIR).

TABLE I. Deposition conditions for BN films.

\begin{tabular}{ll}
\hline \hline Target: & sintered $h$-BN \\
rf input power: & $500 \mathrm{~W}\left(10.8 \mathrm{~W} / \mathrm{cm}^{2}\right)$ \\
Target self-bias: & $-420 \mathrm{~V}$ \\
Substrate self-bias: & $-40 \mathrm{~V}$ and $-80 \mathrm{~V}$ \\
Gas mixture: & $90 \% \mathrm{Ar}, 10 \% \mathrm{~N}_{2}$ \\
Total gas flow: & $3 \mathrm{sccm}$ \\
Pressure: & $\approx 1.5 \times 10^{-1} \mathrm{~Pa}$ \\
Substrate temperature: & $350^{\circ} \mathrm{C}$ \\
Deposition time: & $25 \mathrm{~min}$ \\
\hline \hline
\end{tabular}

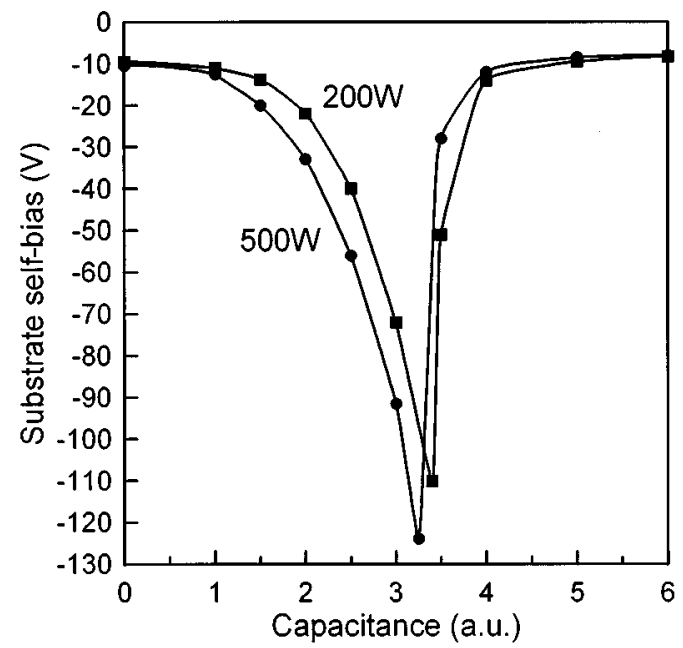

Fig. 2. Dependence of substrate self-bias on tuning network capacitance for an Ar flow of $1.4 \mathrm{sccm}$ and two rf power values. In this experiment the stray capacitance was minimized.

\section{RESULTS}

Figure 2 shows the experimental dependence of substrate bias on tuning network capacitance, for an argon flow of 1.4 sccm and rf power of 200 and $500 \mathrm{~W}$. A sharp minimum (a negative maximum) of substrate bias is obtained when the net inductive reactance of the tuning network resonates with the substrate sheath capacitance, as discussed below. This is accompanied by a visible focusing of the plasma into the region between the target and the substrate. High values of negative substrate bias are limited by the presence of stray capacitance in the system. This effect is clearly observed in Fig. 3, where an additional positive maximum of substrate bias appears, along with a reduction of the negative maximum value, when the stray capacitance is not minimized.

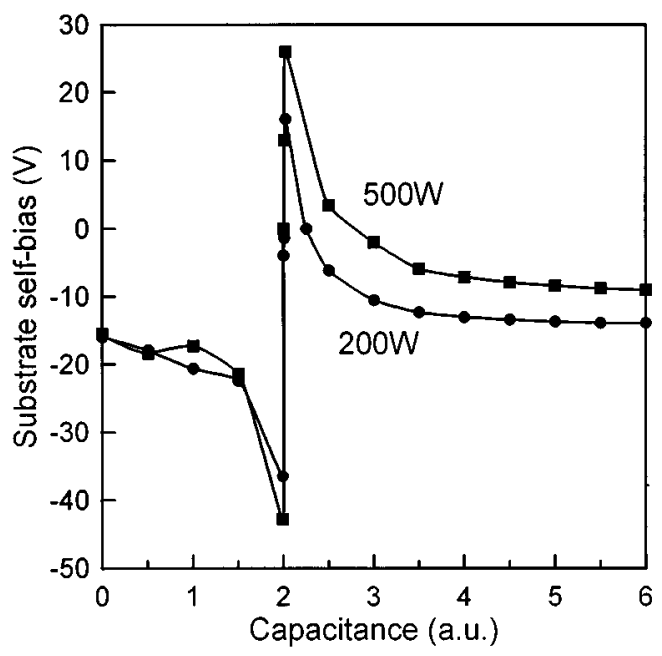

FIG. 3. Substrate self-bias vs tuning network capacitance for two rf power values. The data were collected in a plasma with a $\mathrm{N}_{2}$ flow of $3.7 \mathrm{sccm}$ and an Ar flow of $1.9 \mathrm{sccm}$. In this case, the stray capacitance was not minimized. 


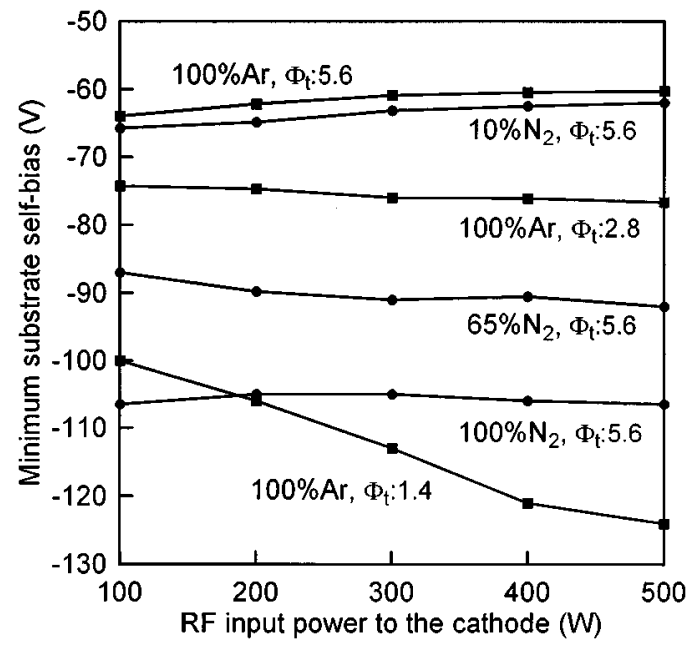

FIG. 4. Maximum negative substrate bias vs rf input power to the cathode for different gas flows and gas compositions. The stray capacitance is here minimized.

Figure 4 shows the dependence of the negative maximum substrate bias on the rf input power to the magnetron cathode for different gas flows and gas compositions. Little dependence of substrate bias on power is observed.

The infrared spectra of two characteristic $\mathrm{BN}$ films are shown in Fig. 5. All the films in this series were deposited in the experimental conditions shown in Table I, with the exception of substrate bias. These spectra were obtained by calculating the ratio of the experimental spectra of the $\mathrm{BN}$ coated substrate to a background scan taken from an uncoated substrate.

\section{DISCUSSION}

The peak in negative substrate bias observed in our rf magnetron sputtering system has a similar behavior to that reported for rf diode sputtering systems. ${ }^{4,8}$ This behavior has

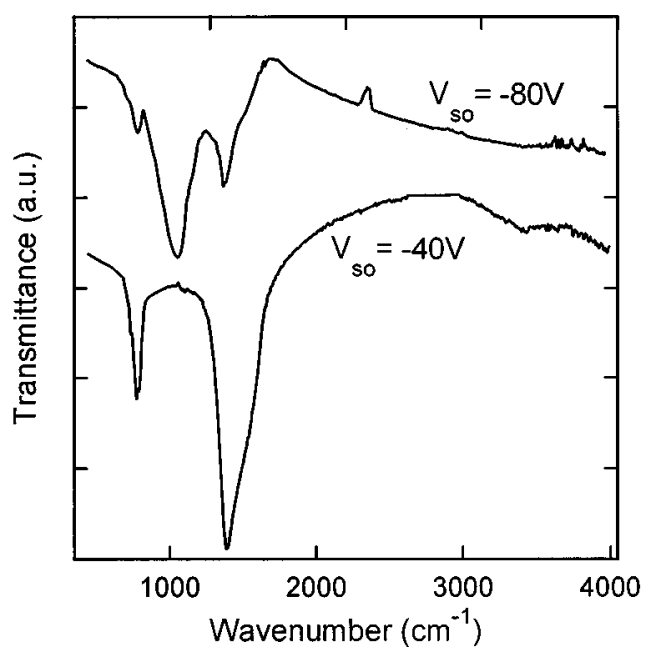

FIG. 5. Infrared spectra of two boron nitride films deposited at different substrate bias $\left(V_{S 0}\right)$. The rest of experimental conditions are shown in Table I.

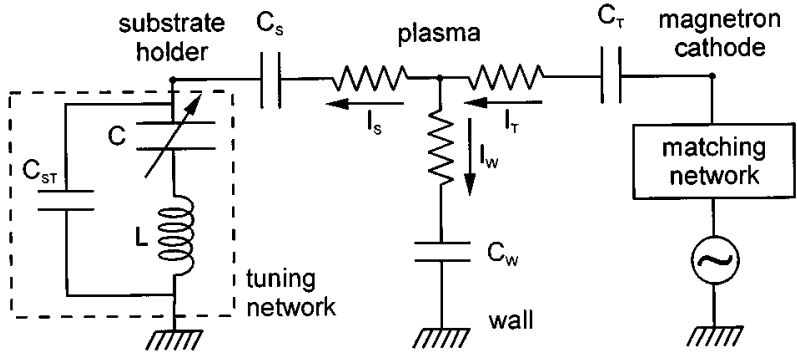

FIG. 6. Electrical model for the experimental system including the substrate tuning network (dashed squared).

been attributed to series resonance between the inductive tuning network and the capacitive substrate sheath., ${ }^{4,8}$

Although a theoretical model of rf sputtering systems has been developed, ${ }^{8}$ it is difficult to apply to other results owing to the lack of explicit solutions to the equations. Here we use a simple model (Fig. 6) to account qualitatively the main features in our system. The plasma boundaries at each of the three electrodes (target, substrate, and wall) are represented by three capacitors. The possible presence of stray capacitance is represented by a capacitor $C_{s t}$, in parallel with the $L-C$ tuning impedance. This equivalent circuit presents a minimum and a maximum of impedance in the path from the plasma to ground through the substrate for the conditions of resonance and antiresonance, respectively. For a fixed value of $L$, the corresponding values of $C$ are:

$$
C_{R}=\frac{1}{L \omega^{2}-\frac{1}{C_{S}+C_{S T}}},
$$

$$
C_{A R}=\frac{1}{L \omega^{2}-\frac{1}{C_{S T}}} .
$$

This model gives a qualitative explanation of our experimental results. The rf current flowing through the plasma (from the cathode) $I_{T}$ is divided into the rf current to wall, $I_{W}$, and the rf current to substrate, $I_{S}$. A maximum of $I_{S}$ is obtained for the condition of resonance, and consequently a maximum of negative bias of the substrate (Figs. 2 and 3), since electrons have a higher mobility than ions. In the same way, either a minimum of the negative bias or a maximum of the positive bias of the substrate is predicted by our model for the condition of antiresonance for a capacitance $C_{A R}>C_{R}$, which is in accordance with our experimental results (Fig. 3). Moreover, as the stray capacitance $C_{S T}$ is reduced, the condition of antiresonance disappears, as observed experimentally (Fig. 2).

Another experimental confirmation of the model is found in the dependence of $C_{R}$ on $\mathrm{rf}$ input power of Fig. 2. As the rf power is increased, the sheath thickness decreases ${ }^{5}$ and so $C_{S}$ increases, which lowers the value of $C_{R}$ [Eq. (1)].

The behavior of different gases shown in Fig. 4 is similar to that observed in unbalanced dc magnetrons. ${ }^{9}$ For a con- 
stant input power, the value of the substrate bias increases with decreasing pressure, as predicted from the general behavior of rf plasmas. ${ }^{5,10}$

The energy of ions reaching the substrate is proportional to the potential $V_{P S}$ of the plasma with respect to the substrate. This energy is calculated by subtracting the substrate bias, $V_{S 0}$, from the plasma potential with respect to ground, $V_{P 0}$. A Langmuir probe ${ }^{11}$ was used to measure $V_{P 0}$, the electron temperature $T_{e}$, and the plasma density $N$. Values of $V_{P 0} \approx 40 \mathrm{~V}, T_{e} \approx 8 \mathrm{eV}$ and $N \approx 5 \times 10^{16} \mathrm{~m}^{-3}$ were obtained for an argon flow of $2.8 \mathrm{sccm}$ and a rf power of 300 W. Note that these results are practically unaffected by changing the substrate bias through the tuning circuit. From these values and the Bohm sheath criterion, the ion flux to the substrate may be estimated, ${ }^{12}$ and we found $\phi_{i}=1.3 \times 10^{20} \mathrm{~m}^{-2} \mathrm{~s}^{-1}$. On the other hand, using the resonance value of $V_{S 0}$ from Fig. 2, the maximum value of $V_{P S}$ for these experimental conditions is $150 \mathrm{~V}$.

Although the deposition conditions necessary for $c$-BN growth are not yet completely understood ${ }^{13,14}$ it is established that ion energy and ion flux are critical in $c$-BN deposition. ${ }^{15}$ The FTIR spectra of Fig. 5 clearly show this effect. All films in this series were deposited under the same ion flux (as deduced from Langmuir measurements) and with different ion energies. Films deposited at ion energy below $80 \mathrm{eV}\left(V_{S 0}=-40 \mathrm{~V}\right)$ show the characteristic peaks of the hexagonal phase $\left(h\right.$-BN) of BN: at $1385 \mathrm{~cm}^{-1}$ (due to inplane $\mathrm{BN}$ mode of vibration) and at $780 \mathrm{~cm}^{-1}$ (due to the out-of-plane $B-N-B$ bending mode). When the ion energy is increased to $120 \mathrm{eV}\left(V_{S 0}=-80 \mathrm{~V}\right)$ the contribution of the $h$-BN decreases, and a strong absorption band at 1060 $\mathrm{cm}^{-1}$ appears, corresponding to the cubic phase contribution $(c-\mathrm{BN})$ in the film. These results, although not yet optimized, are comparable to those obtained by other techniques which require an additional rf source to achieve a controlled substrate bias. ${ }^{16-18}$ On the other hand, when using an additional rf source to increase the negative substrate bias, the additional $\mathrm{rf}$ power supplied to the plasma may increase the plasma density and the associate ion flux, which means that these parameters cannot be controlled independently.

\section{CONCLUSION}

We have shown that the substrate tuned in a rf magnetron systems is a simple and effective method to achieve thin film deposition under controlled ion bombardment. A simple model allows us to interpret the experimental negative peak of the substrate bias as corresponding to a series resonance between the capacitance of the plasma sheath on the substrate and the inductive external inpedance. The presence of stray capacitance causes antiresonance which diminishes the resonance effect. Using the Langmuir probe it has been shown that ion flux and ion energy can be controlled independently. Ion flux is of the order of $10^{20} \mathrm{~m}^{-2} \mathrm{~s}^{-1}$, and ion energy can be varied in the range $30-150 \mathrm{eV}$. This method has been applied successfully to the deposition of BN thin films with a high content of the cubic phase.

\section{ACKNOWLEDGMENT}

This work was supported by the CICYT of Spain under Contract No. MAT93-0298.

${ }^{1}$ O. Auciello and R. Kelly, Ion Bombardment Modification of Surfaces (Fundamentals and Applications) (Elsevier, Amsterdam, Netherlands, 1984), p. 153

${ }^{2}$ D. Li, S. Lopez, Y. W. Chung, M. S. Wong, and W. D. Sproul, J. Vac. Sci. Technol. A 13, 1063 (1995)

${ }^{3}$ S. Reinke, M. Kuhr, and W. Kulisch, Diam. Relat. Mater. 3, 341 (1994).

${ }^{4}$ J. S. Logan, IBM J. Res. Dev. 14, 172 (1970).

${ }^{5}$ S. M. Rossnagel, J. J. Cuomo, and W. D. Westwood, Handbook of Plasma Processing Technology (Noyes, New Jersey, 1990).

${ }^{6}$ N. Savvides and B. Window, J. Vac. Sci. Technol. A 4, 504 (1986).

${ }^{7}$ B. Window and N. Savvides, J. Vac. Sci. Technol. A 4, 196 (1986).

${ }^{8}$ J. H. Keller and W. B. Pennebaker, IBM J. Res. Dev. 23, 3 (1979).

${ }^{9}$ B. Window and N. Savvides, J. Vac. Sci. Technol. A 3, 453 (1986).

${ }^{10}$ A. Grill, Cold Plasma in Materials Fabrication (IEEE, New York, 1994), p. 38.

${ }^{11}$ A. P. Paranjpe, J. P. McVittie, and S. A. Self, J. Appl. Phys. 67, 6718 (1990).

${ }^{12}$ B. Chapman, Glow Discharge Processes (Wiley, New York, 1980).

${ }^{13}$ D. J. Kester, K. S. Ailey, D. J. Lichtenwalner, and R. F. Davis, J. Vac. Sci Technol. A 12, 3074 (1994).

${ }^{14}$ S. Reinke, M. Kuhr, W. Kulisch, and R. Kassing, Diam. Relat. Mater. 4, 272 (1995).

${ }^{15}$ D. J. Kester and R. Messier, J. Appl. Phys. 72, 504 (1992).

${ }^{16}$ W. Gissler, J. Haupt, A. Hoffmann, P. N. Gibson, and D. G. Rickerby, Thin Solid Films 199, 113 (1991).

${ }^{17}$ M. Mieno and T. Yoshida, Surf. Coat. Technol. 52, 87 (1992).

${ }^{18}$ V. Y. Kulikovsky, L. R. Shaginyan, V. M. Vereschaka, and N. G. Hatynenko, Diam. Relat. Mater. 4, 113 (1995) 